\title{
Bridging veins and veins of the brainstem in microvascular decompression surgery for trigeminal neuralgia and hemifacial spasm
}

\author{
Hiroki Toda, MD, PhD, ${ }^{1,2}$ Koichi Iwasaki, MD, PhD, ${ }^{1}$ Naoya Yoshimoto, MD,1 \\ Yoshihito Miki, MD, PhD, ${ }^{1}$ Hirokuni Hashikata, MD, PhD, ${ }^{1}$ Masanori Goto, MD, ${ }^{1}$ and \\ Namiko Nishida, MD, PhD ${ }^{1}$ \\ 'Department of Neurosurgery, Tazuke Kofukai Medical Research Institute and Kitano Hospital, Osaka; and 'Department of \\ Neurosurgery, Fukui Red Cross Hospital, Fukui, Japan
}

OBJECTIVE In microvascular decompression surgery for trigeminal neuralgia and hemifacial spasm, the bridging veins are dissected to provide the surgical corridors, and the veins of the brainstem may be mobilized in cases of venous compression. Strategy and technique in dissecting these veins may affect the surgical outcome. The authors investigated solutions for minimizing venous complications and reviewed the outcome for venous decompression.

METHODS The authors retrospectively reviewed their surgical series of microvascular decompression for trigeminal neuralgia and hemifacial spasm in patients treated between 2005 and 2017. Surgical strategies included preservation of the superior petrosal vein and its tributaries, thorough dissection of the arachnoid sleeve that enveloped these veins, cutting of the inferior petrosal vein over the lower cranial nerves, and mobilization or cutting of the veins of the brainstem that compressed the nerve roots. The authors summarized the patient characteristics, operative findings, and postoperative outcomes according to the vascular compression types as follows: artery alone, artery and vein, and vein alone. They analyzed the data using chi-square and 1-way ANOVA tests.

RESULTS The cohort was composed of 121 patients with trigeminal neuralgia and 205 patients with hemifacial spasm. The superior petrosal vein and its tributaries were preserved with no serious complications in all patients with trigeminal neuralgia. Venous compression alone and arterial and venous compressions were observed in $4 \%$ and $22 \%$, respectively, of the patients with trigeminal neuralgia, and in $1 \%$ and $2 \%$, respectively, of those with hemifacial spasm ( $p<$ 0.0001). In patients with trigeminal neuralgia, $35 \%$ of those with artery and venous compressions and $80 \%$ of those with venous compression alone had atypical neuralgia $(p=0.015)$. The surgical cure and recurrence rates of trigeminal neuralgias with venous compression were $60 \%$ and $20 \%$, respectively, and with arterial and venous compressions the rates were $92 \%$ and $12 \%$, respectively $(p<0.0001, p=0.04)$. In patients with hemifacial spasm who had arterial and venous compressions, their recurrence rate was $60 \%$, and that was significantly higher compared to other compression types ( $p$ $=0.0008$ ).

CONCLUSIONS Dissection of the arachnoid sleeve that envelops the superior petrosal vein may help to reduce venous complications in surgery for trigeminal neuralgia. Venous compression may correlate with worse prognosis even with thorough decompression, in both trigeminal neuralgia and hemifacial spasm.

https://thejns.org/doi/abs/10.3171/2018.4.FOCUS18122

KEYWORDS microvascular decompression surgery; veins; arachnoid; trigeminal neuralgia; hemifacial spasm

$\mathrm{T}$ wo types of posterior fossa veins are involved in microvascular decompression surgery, namely, the bridging veins and veins of the brainstem..$^{20}$ Bridging veins often traverse the surgical corridors to the trigeminal and facial nerves. ${ }^{19}$ Veins of the brainstem may sometimes compress the trigeminal and facial nerve roots and cause trigeminal neuralgias, $4,8,10,11,15,17$ and hemifacial spasms, ${ }^{2,6,7}$ respectively. Therefore, venous dissection is essential in microvascular decompression surgery, but the dissection of these veins may cause severe surgical com-

ABBREVIATIONS BNI = Barrow Neurological Institute; TOF = time of flight; T2-DRIVE = T2-weighted-driven equilibrium .

SUBMITTED March 1, 2018. ACCEPTED April 4, 2018.

INCLUDE WHEN CITING DOI: 10.3171/2018.4.FOCUS18122. 
plications., ${ }^{3,12,13,22}$ To elucidate safe and effective methods of venous dissection, we reviewed our surgical series of microvascular decompressions for trigeminal neuralgia and hemifacial spasm. We preserved the superior petrosal vein and its tributaries in the surgeries for trigeminal neuralgia, whereas we cut the inferior petrosal vein near the accessory nerve early in the surgeries for hemifacial spasm. We illustrate the usefulness of cutting arachnoid membrane that enveloped the superior petrosal vein to preserve this vein. This arachnoid dissection enables further dissection around the trigeminal nerve root to be performed safely. We also focus on the clinical importance of venous compression to the trigeminal $1^{4,8,10,11,15,17}$ and facial nerve roots. ${ }^{2,6,7}$ We summarize the cases with venous compression and their long-term outcomes after microvascular decompression surgery.

\section{Methods}

\section{Patient Populations and Preoperative Examinations}

A review of our institutional clinical database identified patients who underwent microvascular decompression for trigeminal neuralgias and hemifacial spasms between 2005 and 2017. The recurrent cases were excluded from the current study, and our institutional review board approved the study. We retrospectively analyzed the medical records and operative findings of these patients. The preoperative evaluations included neurological assessment and MRI with 3D time-of-flight (TOF) and T2-weighteddriven equilibrium (T2-DRIVE) sequences. ${ }^{21}$ As for trigeminal neuralgia, the facial pain was categorized into typical (type 1) and atypical (type 2) neuralgia. ${ }^{1,17}$ Images of 3D TOF were used to follow arteries, and those of 3D T2-DRIVE were used to evaluate the roots of the cranial nerves and artery and venous vessels. ${ }^{21}$ We defined the radiological vascular compression as the presence of distortion or indentation of the nerve root by the vessels. Comparison of the 3D TOF artery and T2-DRIVE artery and vein images allowed visualization of the venous courses and the presence of venous compression. A vein slightly distorting the nerve root and a vein crossing over the nerve root were regarded as the presence of radiological venous compression. Patients with no radiological vascular compression were excluded from this study.

\section{Surgical Procedures}

All patients underwent microvascular decompression surgery using a surgical technique that was modified from previous reports. ${ }^{9,16,19,21}$ In surgeries for trigeminal neuralgias, the superior petrosal vein and its tributaries were preserved as much as possible. After confirmation that arachnoid sleeve enveloped the superior petrosal vein (Fig. 1A and B, Video 1), we cut this arachnoid sleeve, using slightly round-tipped microscissors and a 3-Fr suction tube (Fig. 1C-E), in all 105 cases of trigeminal neuralgias treated surgically since mid-2008.

VIDEO 1. Our surgical technique of arachnoid dissection to preserve the superior petrosal vein and its tributaries for a patient with left trigeminal neuralgia is shown. Copyright Hiroki Toda. Published with permission. Click here to view.

The superior petrosal vein and its tributaries were thor- oughly exposed (Fig. 1F), and the trigeminal nerve root and its compressing vessels were observed (Fig. 1G). Further neurovascular dissection was performed through the corridors that were lined by these veins, the dural surface, and the cerebellum (Fig. 1H). In the surgeries for hemifacial spasms, the inferior petrosal vein near the accessory nerve was usually coagulated and cut before dissection of the lower cranial nerves, to avoid hemorrhages from this vein. For both trigeminal neuralgia and hemifacial spasms, the arachnoid between the nerve roots and offending vessels was cut, to mobilize the offending vessels from the nerve roots. The presence of vascular compression was confirmed by vascular contact with mild indentation on the nerve root (indentation type), distortion of the nerve root (distortion type), or vascular groove on the nerve root (groove type). ${ }^{4}$ Venous compression was resolved by moving the offending vein from the nerve root or by cutting it. The mobilized vessels were fixed to the dura mater or adjacent structures by using a small amount of fibrin glue and oxidized cellulose. We have summarized the operative findings regarding the offending vessels and the compressed nerve roots.

\section{Postoperative Evaluations}

All patients were hospitalized for 7 days or more, to observe immediate postoperative courses. All but 1 patient with a cardiac pacemaker postoperatively underwent MRI. The postoperative evaluations included surgical outcomes and complications. The outcome indices at 12 months or more after surgery were as follows: the Barrow Neurological Institute (BNI) pain intensity score and postoperative dose of carbamazepine for trigeminal neuralgias, and the improvement of facial twitches for hemifacial spasms. Complications included postoperative events with prolonged hospital stays, deteriorated neurological functions, and additional examinations.

\section{Statistical Analysis}

All statistical analyses were performed using IBM SPSS Statistics for Windows, version 24.0 (IBM Corp.). The categorical patient characteristic data were assessed by chi-square tests. The numerical data were compared using a 1-way ANOVA, with Tukey's multiple comparison tests; $p$ values less than 0.05 were considered statistically significant.

\section{Results}

\section{Management of Bridging Veins in Trigeminal Neuralgia and Hemifacial Spasm Surgeries: The Superior and Inferior Petrosal Veins}

The study cohort was composed of 121 patients with trigeminal neuralgia (46 men, 75 women; mean age 64 years, range $29-89$ years; 85 with typical, 36 with atypical neuralgia; median follow-up period of 63 months, range 5-147 months; Table 1) and 205 patients with hemifacial spasms (64 men, 141 women; mean age 53 years, range 22-77 years; median follow-up period of 63 months, range 6-120 months; Table 2). In 16 patients with trigeminal neuralgias who underwent surgery between 2005 and 2008 , the arachnoid sleeve that enveloped the superior pe- 

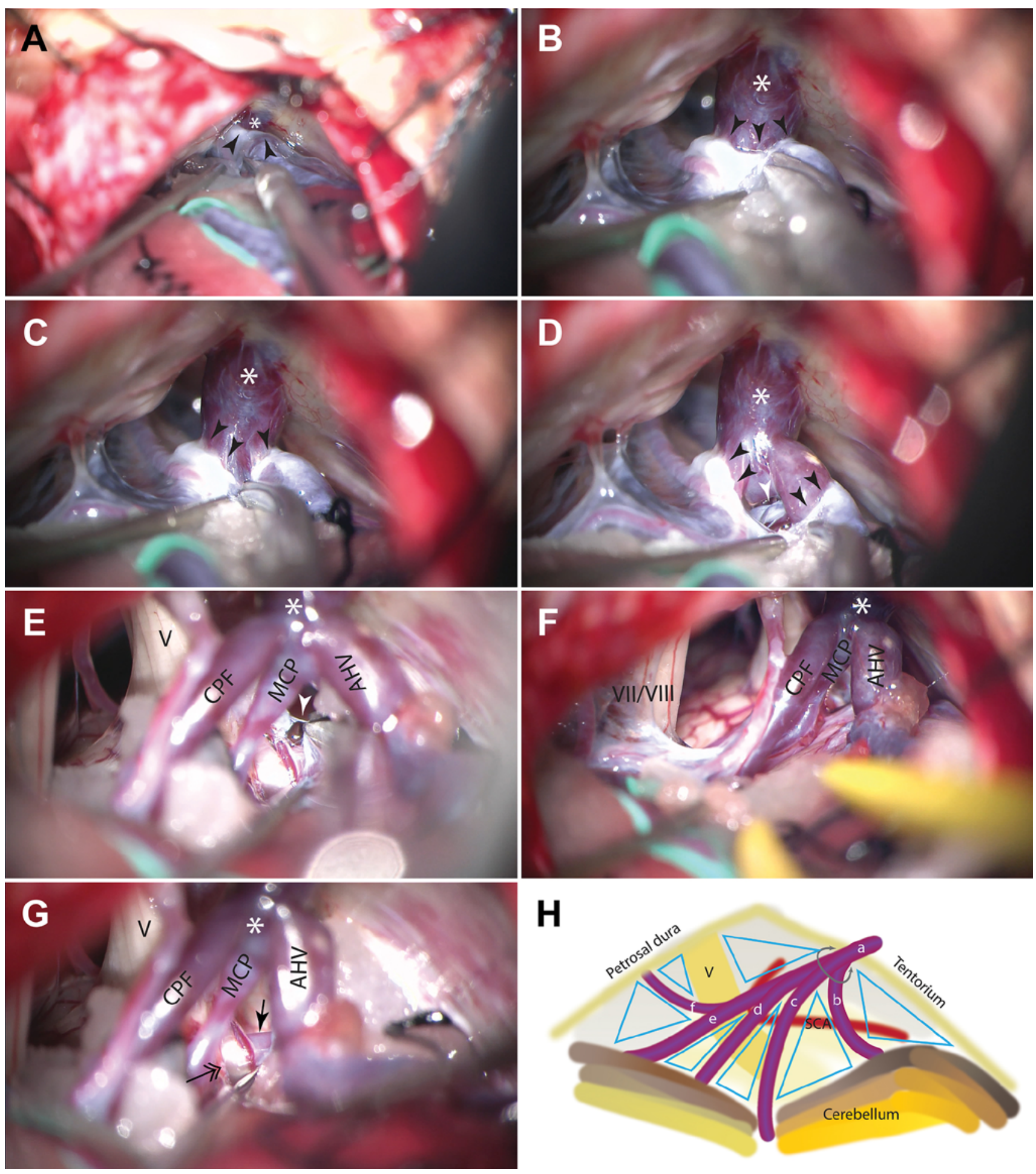

FIG. 1. A: Intraoperative photograph of the microvascular decompression for left trigeminal neuralgia, showing the arachnoid (arrowheads) over the superior petrosal vein (SPV; asterisk). B: A magnified view showing the arachnoid sleeve (arrowheads) that envelops the SPV (asterisk). C: Incision of the arachnoid sleeve (arrowheads) exposing the tributary veins to the SPV (aster-

isk). D: Cutting the front end of the arachnoid sleeve (black arrowheads) in front of the SPV (asterisk) exposes its back end (white arrowhead) behind the SPV. E: Cutting the back end of the arachnoid sleeve (arrowhead). F: The SPV (asterisk) and its tributaries are dissected from their arachnoid sleeve. G: Through the spaces between AHV, MCP, and CPF, compressed trigeminal nerve root (2-headed arrow) by the superior cerebellar artery (SCA; arrow) is shown. H: Triangular spaces for observation of the trigeminal nerve root $(\mathrm{V})$ and the SCA. The SPV $(a)$ is covered by the arachnoid sleeve (circular arrow): $b$, pontotrigeminal vein; $c$, anterior hemispheric vein; $d$, vein of cerebellopontine fissure; $e$, vein of middle cerebellar peduncle; $f$, transverse pontine vein. AHV = anterior hemispheric vein; $\mathrm{CPF}=$ vein of cerebellopontine fissure; $\mathrm{MCP}=$ vein of middle cerebellar peduncle; $\mathrm{VII} / \mathrm{VIII}=$ facial nerve/vestibulocochlear nerve. Copyright Hiroki Toda (panel H). Published with permission. 
TABLE 1. Trigeminal neuralgias: characteristics, operative findings, and treatment outcomes in 121 patients who underwent microvascular decompression

\begin{tabular}{|c|c|c|c|c|c|}
\hline \multirow[b]{3}{*}{ Variable } & \multicolumn{4}{|c|}{ Offending Vessel Group } & \multirow[b]{3}{*}{$\mathrm{p}$ Value } \\
\hline & \multirow[b]{2}{*}{ Artery } & \multicolumn{2}{|c|}{ Vein } & \multirow[b]{2}{*}{ None } & \\
\hline & & Artery \& Vein & Vein Only & & \\
\hline \multicolumn{6}{|l|}{ Preop evaluation } \\
\hline Patients & $87(72)$ & $26(22)$ & $5(4)$ & $3(2)$ & \\
\hline \multicolumn{6}{|l|}{ Sex } \\
\hline Female & $50(57)$ & $19(73)$ & $5(100)$ & $1(33)$ & 0.10 \\
\hline Male & $37(42)$ & $7(27)$ & $0(0)$ & $2(67)$ & \\
\hline Mean age in yrs, range & $64.1,29-89$ & $64.3,32-85$ & $62.0,60-65$ & $64.3,56-78$ & 0.98 \\
\hline \multicolumn{6}{|l|}{ Affected side } \\
\hline Rt & $50(57)$ & $16(62)$ & $4(80)$ & $1(33)$ & 0.60 \\
\hline $\mathrm{Lt}$ & $37(42)$ & $10(38)$ & $1(20)$ & $2(67)$ & \\
\hline \multicolumn{6}{|l|}{ Neuralgia type } \\
\hline Typical: type 1 & $67(77)$ & $17(65)$ & $1(20)$ & $0(0)$ & 0.015 \\
\hline Atypical: type 2 & $20(23)$ & $9(35)$ & $4(80)$ & $3(100)$ & \\
\hline \multicolumn{6}{|l|}{ Neuralgia location } \\
\hline V1 & $17(20)$ & $4(15)$ & $0(0)$ & $0(0)$ & 0.64 \\
\hline V2 & $59(68)$ & $19(73)$ & $2(40)$ & $2(67)$ & \\
\hline V3 & $47(54)$ & $17(65)$ & $5(100)$ & $2(67)$ & \\
\hline Patients with CBZ & $82(95)$ & $24(92)$ & $4(80)$ & $3(100)$ & 0.61 \\
\hline Median CBZ dose in $\mathrm{mg}$, range & $400,100-1200$ & $400,200-800$ & $500,400-600$ & $400,300-900$ & 0.41 \\
\hline Median follow-up in mos, range & $63,5-147$ & $55,24-147$ & $61,5-70$ & $45,33-102$ & 0.20 \\
\hline \multicolumn{6}{|l|}{ Operative findings } \\
\hline \multicolumn{6}{|l|}{ Offending vessel } \\
\hline SCA & $81(93)$ & $25(96)$ & & & \\
\hline AICA & $23(27)$ & $4(15)$ & & & \\
\hline TPV & & $14(54)$ & $4(80)$ & & \\
\hline PTV & & $8(31)$ & $1(20)$ & & \\
\hline Others & $5(5)^{*}$ & $5(19) \dagger$ & $2(40) \dagger$ & & \\
\hline Compression pattern & & & & & $<0.0001$ \\
\hline Indentation & $8(9)$ & $13(50)$ & $3(60)$ & & \\
\hline Distortion & $41(47)$ & $4(15)$ & $1(20)$ & & \\
\hline Groove & $38(44)$ & $7(27)$ & $1(20)$ & & \\
\hline Intraneural vein & NA & $2(8)$ & $0(0)$ & & \\
\hline Compression site & & & & & $<0.0001$ \\
\hline Root entry zone & $79(91)$ & $3(12)$ & $1(20)$ & & \\
\hline Cisternal & $31(36)$ & $22(85)$ & $4(80)$ & & \\
\hline Porus of Meckel's cave & $6(7)$ & $8(31)$ & $2(40)$ & & \\
\hline Surgical complications & $4(5) \ddagger$ & $1(4) \S$ & $1(20) \pi$ & & 0.45 \\
\hline \multicolumn{6}{|l|}{ Postop status } \\
\hline Median BNI grade, range & I, I-III & I, I-III & I, I-III & IV, I-IV & \\
\hline BNI grade I & $72(83)$ & $24(92)$ & $3(60)$ & $1(33)$ & $<0.0001$ \\
\hline Median CBZ dose in $\mathrm{mg}$, range & $0,0-800$ & $0,0-300$ & $0,0-400$ & $400,0-900$ & $<0.0001$ \\
\hline Recurrence & $3(3.5)$ & $3(12)$ & $1(20)$ & $0(0)$ & 0.04 \\
\hline
\end{tabular}

$\mathrm{AICA}=$ anterior inferior cerebellar artery; $\mathrm{BA}=$ basilar artery; $\mathrm{CBZ}=$ carbamazepine; $\mathrm{MCA}=$ middle cerebral artery; $\mathrm{NA}=$ not applicable; $\mathrm{PICA}=$ posterior inferior cerebellar artery; PTV = pontotrigeminal vein; SCA = superior cerebellar artery; TPV = transverse pontine vein; VA = vertebral artery.

Unless otherwise indicated, the values are expressed as the number of patients (\%).

* 2 PICAs, 1 persistent trigeminal artery variant, $1 \mathrm{BA}$, and $1 \mathrm{VA}$.

$\dagger$ Other veins.

$\ddagger 2$ subdural effusion or hematoma, 1 deep venous thrombosis, 1 transient facial paresthesia.

$\S 1$ cerebral infarction due to MCA stenosis.

If 1 cerebellar hemorrhage. 
TABLE 2. Hemifacial spasms: characteristics, operative findings, and treatment outcomes in 205 patients who underwent microvascular decompression

\begin{tabular}{|c|c|c|c|c|}
\hline \multirow[b]{3}{*}{ Variable } & \multicolumn{3}{|c|}{ Offending Vessel Group } & \multirow[b]{3}{*}{$\begin{array}{c}p \\
\text { Value }\end{array}$} \\
\hline & \multirow[b]{2}{*}{ Artery } & \multicolumn{2}{|c|}{ Vein } & \\
\hline & & $\begin{array}{l}\text { Artery } \\
\& \text { Vein }\end{array}$ & Vein Only & \\
\hline \multicolumn{5}{|l|}{ Preop evaluation } \\
\hline Patients & $198(97)$ & $5(2)$ & $2(1)$ & \\
\hline \multicolumn{5}{|l|}{ Sex } \\
\hline Female & $136(69)$ & $4(80)$ & $1(50)$ & 0.83 \\
\hline Male & $62(31)$ & $1(20)$ & $1(50)$ & \\
\hline Mean age in yrs, range & $53,22-77$ & $59,36-69$ & $33,27-39$ & 0.98 \\
\hline \multicolumn{5}{|l|}{ Affected side } \\
\hline Rt & $86(43)$ & $3(60)$ & $0(0)$ & 0.47 \\
\hline Lt & $112(57)$ & $2(40)$ & $2(100)$ & \\
\hline \multicolumn{5}{|l|}{ Operative findings } \\
\hline \multicolumn{5}{|l|}{ Offending vessel } \\
\hline AICA & $157(79)$ & $3(60)^{*}$ & $2(100)$ & \\
\hline PICA & $71(36)$ & $2(40) \dagger$ & & \\
\hline VA & $79(40)$ & & & \\
\hline \multicolumn{5}{|l|}{ Postop status } \\
\hline Cure & $173(87)$ & $4(80)$ & $2(100)$ & 0.61 \\
\hline $\begin{array}{l}\text { Recurrence in long } \\
\text { term }\end{array}$ & $6(3)$ & $3(60) \ddagger$ & $0(0)$ & 0.0008 \\
\hline
\end{tabular}

Unless otherwise indicated, the values are expressed as the number of patients $(\%)$.

* AICA plus veins.

$\dagger$ PICA plus veins.

$\ddagger 2$ of 3 patients underwent repeat microvascular decompression and their hemifacial spasms were cured.

trosal vein was not cut routinely. There were 2 patients (13\%) who had bleeding from the junction of the superior petrosal vein into the superior petrosal sinus. These patients' bleeding was controlled with saline irrigation and use of oxidized cellulose, and the patients' superior petrosal veins were preserved. Since mid-2008, 105 patients underwent microvascular decompression surgery with cutting of the arachnoid sleeve. One patient (1\%) had bleeding from a superficial vein during dissection. Preservation of the superior petrosal veins was possible in all cases in our series (Fig. 1A-G, Video 1). Two patients (2\%) had duplicated superior petrosal veins; both duplicates were preserved in the first patient, and one of the duplicates was cut and the other was preserved in the second patient. The sacrificed veins were as follows: pontotrigeminal veins in $4(4 \%)$, transverse pontine vein in $2(2 \%)$, and unnamed small veins in 15 patients $(14 \%)$.

One patient (1\%) whose veins had been preserved during surgery experienced intraoperative cerebellar swelling. In this patient, venous compression from the transverse pontine vein and the vein of the cerebellopontine fissure was found and decompressed by mobilizing these veins, and none of the veins were sacrificed. However, the cerebellum had been retracted excessively in the early phase of the surgery to release the CSF after dural opening. The patient temporarily experienced a cerebellar limb ataxia in the affected side; the ataxia disappeared at discharge 1 month after the surgery.

In the other 104 cases (99\%) with cutting of the arachnoid sleeve, there were no cases with venous congestion. Postoperative MRI studies of these patients did not show venous congestion or venous hemorrhages, except in 1 patient with cerebellar swelling, as reported above.

In the surgeries for hemifacial spasms, the inferior petrosal vein near the accessory nerve was cut, regardless of its size. Sacrifice of the inferior petrosal vein enabled a wide opening of the cerebellomedullary fissure. Postoperative MRI studies of these patients with hemifacial spasm showed minor cerebellar contusions along the surgical trajectories in 2 patients (1\%) and no venous congestion or intracerebellar hemorrhages.

\section{Cases With Venous Compression of the Trigeminal Nerve Root and Their Long-Term Outcomes}

Among the 121 patients with trigeminal neuralgia, we intraoperatively confirmed that 31 (26\%) had veininvolved compressions to the trigeminal nerve root (Table 1). Venous compression alone was found in 5 patients $(4 \%$, Fig. 2A and B), and both arterial and venous compressions in 26 patients (22\%, Fig. 2C-F). Arterial compression alone was confirmed in 87 patients (72\%), and no arterial or venous compression was found in 3 patients $(2 \%)$. Atypical trigeminal neuralgias were seen in all 3 patients with no compression, in 4 of 5 patients (80\%) with venous compression alone, in 9 of 26 patients (35\%) with arterial and venous compressions, and in 20 of 87 patients (23\%) with arterial compression alone. A chi-square analysis revealed significant correlations between vein-involved compression and atypical neuralgia $\left(\chi^{2}=8.36 ; \mathrm{p}=0.015\right)$.

Preoperative 3D T2-DRIVE and 3D TOF imaging could identify venous compression accurately in 18 of 26 patients $(69 \%)$ with arterial and venous compressions and in 4 of 5 patients $(80 \%)$ with venous compression alone, whereas arterial compression was predicted accurately in all 87 patients with arterial compression alone. The positive and negative prediction values of our preoperative MRI studies for identifying venous compression were $88 \%$ and $90 \%$, respectively, and their sensitivity and specificity for venous compression were $71 \%$ and $96 \%$, respectively.

The most commonly observed compressing vein was the transverse pontine vein, followed by the pontotrigeminal vein (Table 1). Other causative veins were the veins of the cerebellopontine fissure and of the middle cerebellar peduncle. In patients with arterial and venous compressions and with venous compression alone, the indentation type was the most commonly observed and the distortion and groove types were infrequently observed, compared to those in the arterial compression alone group $\left(\chi^{2}=\right.$ 28.7; $\mathrm{p}<0.01$ ). In addition, the venous compression was commonly found at the cisternal portion of the trigeminal nerve and near Meckel's cave rather than at the root entry zone of the trigeminal nerve $\left(\chi^{2}=42.5 ; p<0.01\right)$.

Postoperatively, $60 \%$ of the patients with venous compression had a BNI score of grade I at 12 months after surgery, whereas $92 \%$ of the patients with arterial and venous 


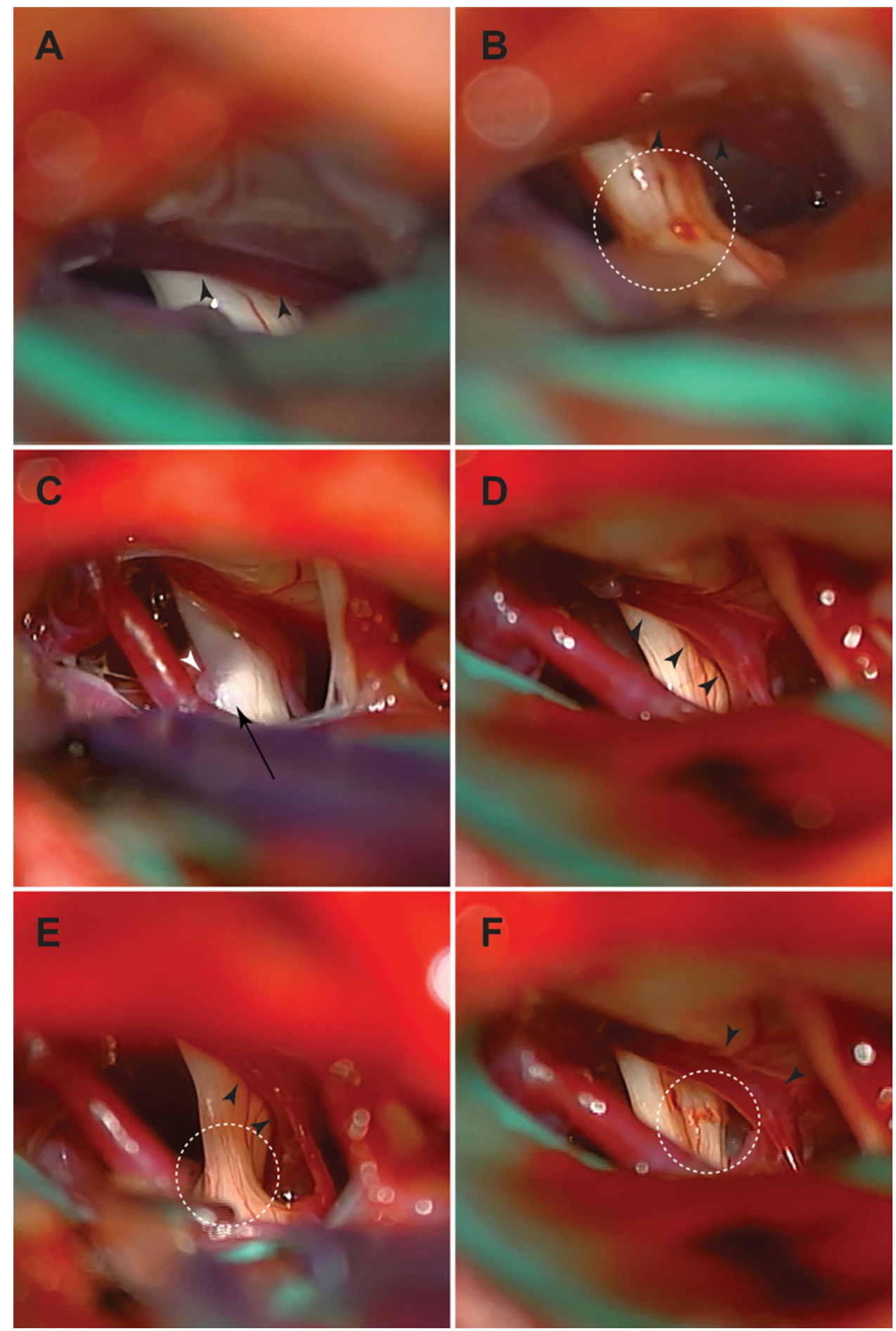

FIG. 2. A and B: Intraoperative photographs showing trigeminal nerve compression by the transverse pontine vein (TPV; arrowheads). After moving the TPV, the compression on the nerve root is observed (dotted circle). C-F: Intraoperative photographs show that the right trigeminal nerve is compressed between the superior cerebellar artery (SCA) and the TPV. C: The SCA (arrowhead) compresses the nerve root (arrow). D: The TPV (arrowheads) compresses the nerve root near Meckel's cave. E: Mobilization of the SCA decompresses the nerve root (dotted circle), while the TPV (arrowheads) remains. F: Dissection and mobilization of the TPV (arrowheads) decompresses the nerve root (dotted circle).

compressions had a BNI grade of I ( $p<0.0001$, Table 1). All 3 patients who had a postoperative BNI grade of III or worse in the vein-related compression groups had atypi- cal trigeminal neuralgia preoperatively. The postoperative dose of carbamazepine also significantly differed according to vascular compression types, as determined using 

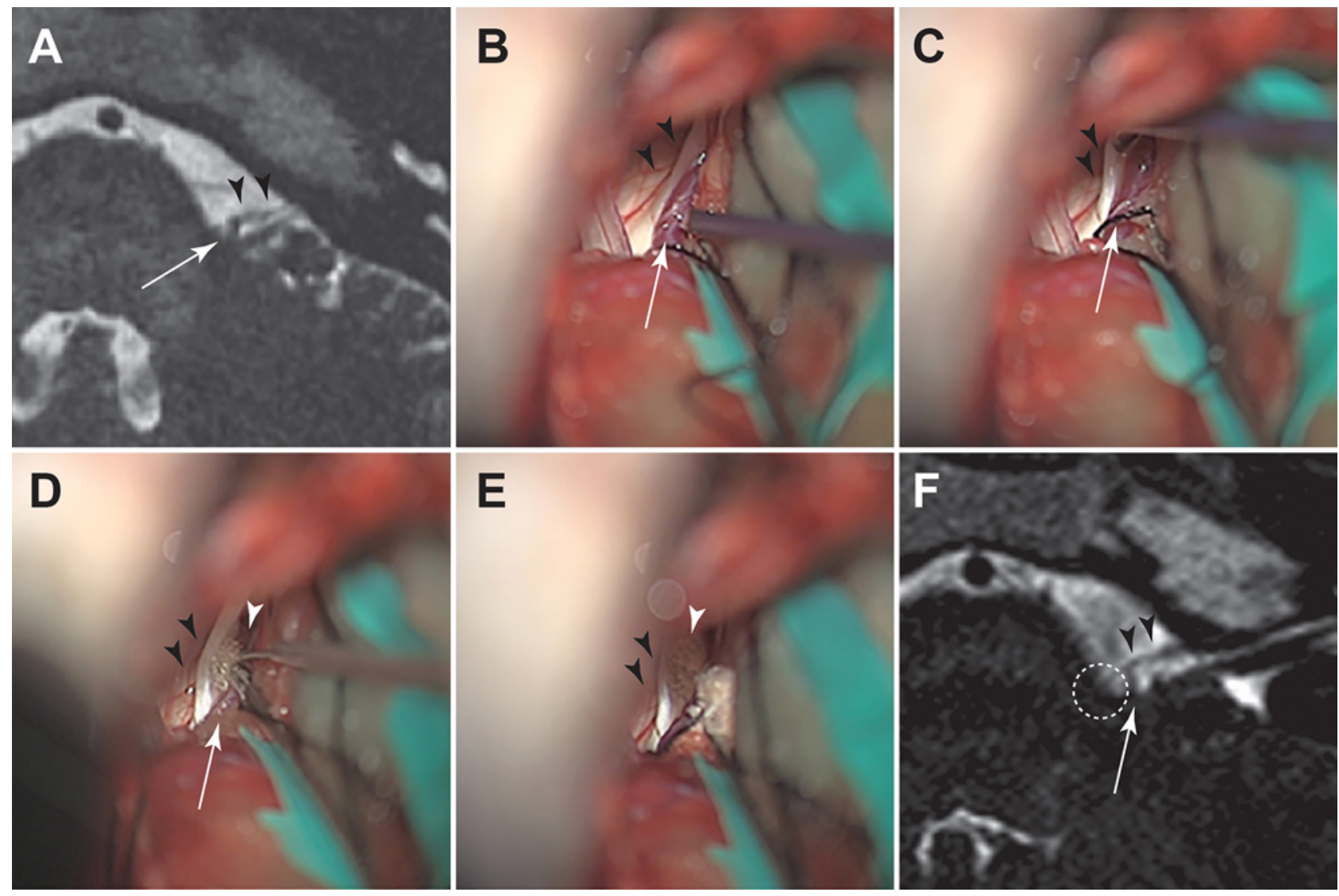

FIG. 3. A: A preoperative MR image predicts the vessel (arrow) between the facial nerve (arrowheads) and the cochlear nerve. B-E: Intraoperative photographs show that the vein of the middle cerebellar peduncle (MCP; arrow) runs between the facial nerve (arrowheads) and the cochlear nerve (B); mobilization of the facial nerve makes a space (C) for insertion of a prosthesis (white arrowhead, D) between the facial nerve and MCP (E). F: A postoperative MR image shows a space at the facial nerve root exit zone (dotted circle). The offending vessel (arrow) and the facial nerve (arrowheads) are shown.

a 1-way ANOVA with Tukey's multiple comparison tests $(\mathrm{F}(3,117)=11.55 ; \mathrm{p}<0.0001)$. Postoperative complications included a cerebral infarction, which was due to comorbid middle cerebral artery stenosis, in 1 patient with arterial and venous compressions, and a cerebellar hemorrhage in 1 patient with venous compression, as described above. In addition, patients with venous compression experienced neuralgias that resolved gradually 3-4 months after surgery. Furthermore, long-term follow-up confirmed that neuralgias recurred in 1 patient (20\%) with venous compression alone and in 3 patients (12\%) with arterial and venous compressions, and that recurrences were seen in 3 patients $(3 \%)$ with arterial compression alone $(\mathrm{p}=0.04)$.

\section{Veins in Microvascular Decompression Surgery for Hemifacial Spasms}

The venous system was less involved in the surgeries for hemifacial spasm compared to surgeries for trigeminal neuralgia. The inferior petrosal vein near the accessory nerve was coagulated and cut for further dissection of the lower cranial nerves. Cutting one of the inferior petrosal veins caused no venous complications, whereas cerebellar retraction before the cutting of these veins caused venous bleeding in 3 cases (1\%), which was controlled with saline irrigation and use of oxidized cellulose. Venous compression of the facial nerve root was observed in 7 patients (3\%, Table 2): venous compression alone in 2 (1\%, Fig. 3), and both arterial and venous compressions in 5 patients (2\%). These vein-involved compressions were significantly rare in hemifacial spasm, compared to that in trigeminal neuralgia $(\mathrm{p}<0.0001)$. While the cure rates of venous compressions $(80 \%-100 \%)$ were comparable to those of arterial compression (87\%), significantly frequent longterm recurrences occurred in cases with arterial and venous compressions $(60 \%)$ compared to those with arterial compression alone (3\%) and those with venous compression alone $(0 \% ; \mathrm{p}=0.0008)$.

\section{Discussion}

Our study may provide some insights into vein-related issues in microvascular decompression surgery for trigeminal neuralgia and hemifacial spasm. A frequently discussed issue is whether the superior petrosal vein should be sacrificed or preserved. $3,12,13,18,22$ There is conflicting literature on this issue. ${ }^{3,12,13,18,22}$ Sacrificing the superior petrosal vein is safe in most cases, ${ }^{16,18}$ and a cerebellar swelling can occur even if the veins are preserved, as seen in our case; however, severe and dangerous venous congestion may oc- 
cur after sacrificing the superior petrosal vein..$^{3,12,13,22}$ The risk of venous congestion can be less than $0.5 \% 16,18$ and as high as $4.8 \% .^{12}$ Liebelt et al. concluded that sacrifice should be limited to cases where it is absolutely necessary. Although there are several studies in which investigators have estimated the extent of the drainage and collateral flow of the superior petrosal vein, ${ }^{14,19,20}$ it has been difficult to accurately estimate the risk of venous congestion in each case. Therefore, we have preserved the superior petrosal vein and its tributaries in microvascular decompression surgeries for trigeminal neuralgia. ${ }^{21}$ Because our study was not a comparative study to validate a specific surgical strategy, we described several surgical nuances that could be used to preserve the superior petrosal vein and its tributaries in microvascular decompression surgery.

We highlighted the usefulness of cutting the arachnoid sleeve that envelops the superior petrosal vein before dissecting the venous structures. Cutting and opening of the arachnoid sleeve can expose the superior petrosal vein and its tributaries. By thoroughly removing the arachnoid from these vessels, the vascular structure can be mobilized during further dissection of the nerve root and the offending artery and venous vessels. Although preserving the venous system may make the surgery slightly more complex, Dumot et al. have reported a similar surgical strategy with excellent surgical results. ${ }^{4}$ We can perform wide and complete surgical manipulations of the offending vessels, using multiple surgical corridors, and preserve the venous flow of the cerebellum and brainstem (Fig. 1H, Video 1).

Venous compression is another vein-related issue, especially in trigeminal neuralgia., ${ }^{4,810,11,15,17}$ This venous compression has been observed in $4.3 \%-25.7 \%$ of the patients with trigeminal neuralgia who underwent microvascular decompression surgery. ${ }^{4,8,10,15,17}$ The surgical outcome was good or excellent in 70.6\%-100\% of these patients, $4,8,10,11,15$ and recurrence was observed in $0 \%-36.4 \%$ of them. . $^{4,8,10,14}$ Compared to those with arterial compression, the patients with venous compression may have relatively less pain improvement and higher recurrence rates, although some have reported excellent outcomes in those with venous compression. ${ }^{10}$ Venous compression is more frequently seen in patients with atypical (type 2) neuralgia than in those with typical (type 1) disease, ${ }^{17}$ and surgical outcomes of patients with atypical disease are inferior to those of patients with typical neuralgia. ${ }^{8,11,17}$ Therefore, patients with atypical neuralgia with venous compression may have a poor prognosis after the surgical treatment. Venous compression is also characterized by the presence of a vein running over the nerve root at its cisternal portion or at the porus of Meckel's cave., ${ }^{4,5,15}$ This characteristic venous neurovascular conflict is contrasted with the arterial neurovascular conflict that is typically caused by an arterial loop at the root exit zone of the trigeminal nerve. ${ }^{16}$ These anatomical differences between venous and arterial compressions may explain the characteristics of trigeminal neuralgia due to venous compression.

The operative issues regarding venous compression are its resolution and the discrimination of venous compression from venous contacts. To resolve the venous compression, offending veins needs to be mobilized or sacrificed.
Considering the anastomotic venous network in the posterior fossa, ${ }^{19,20}$ obliteration of the transverse pontine and pontotrigeminal veins can be safe, whereas obliteration of the veins of the cerebellopontine fissure and of the middle cerebellar peduncle may be unsafe. ${ }^{22}$ We also emphasized the arachnoid dissection between the trigeminal nerve and vessels, including both arteries and veins, and moved these vessels away from the nerve root without the use of prostheses. We emphasize again the importance of complete arachnoid dissection between the nerves and vessels from the root exit zone of the brainstem (Fig. 2E) to the porus of Meckel's cave (Fig. 2F). ${ }^{4}$ However, even with thorough vascular decompression, the relatively low cure rates and the risk of recurrence remain controversial issues in patients with venous compression. The discrimination of venous compression from venous contacts can be difficult at times, and it is often difficult to predict the presence of the venous neurovascular conflict preoperatively. ${ }^{23}$ The discrimination of venous contacts from venous compression can be difficult even during the operation. Dumot and Sindou classified venous compression from mild to severe by referring to the impact of vascular compression on the nerve root. ${ }^{5}$ Further improvement of preoperative neuroimaging studies to predict the severity of venous compression may improve the surgical outcomes in patients with venous compression in the future.

In patients with hemifacial spasm, the importance of the venous system in microvascular decompression may be less. Sacrificing the inferior petrosal vein near the accessory nerve is an essential step to approach the lower cranial nerves. ${ }^{9}$ Compared to the superior petrosal vein, cutting the inferior petrosal vein near the accessory nerve root has been safe and effective in our study. Whereas the superior petrosal vein is the largest vein in the posterior fossa and drains the large areas of the cerebellar hemisphere through the petrosal vein group,,$^{14,19,20}$ the inferior petrosal vein near the accessory nerve is a small and short vein and there are collateral flows to other bridging veins through the lateral medullary vein and the veins of the inferior and middle cerebellar peduncles. ${ }^{19,20}$ In patients with a very large inferior petrosal vein near the accessory nerve, this vein may be dissected and preserved instead of sacrificed.

Venous compression to the root of the facial nerve is rare in hemifacial spasms. ${ }^{2,6,7}$ The veins of the middle cerebellar peduncle and of the pontomedullary sulcus are the common veins near the facial nerve root. ${ }^{19,20}$ Especially, the vein of the middle cerebellar peduncle often runs between the facial and auditory nerves..$^{19}$ Therefore, manipulations such as coagulation or cutting of these veins may injure these nerves. We adopted the insertion of a polyurethane sponge between the facial nerve and the compressing vein in 1 case (Fig. 3B-E). That patient has been free from recurrence for 5 years after surgery. Thus, the use of prosthesis insertions can be an option when the mobilization and cutting of the offending vessels have risks of complications. Similar to findings in patients with trigeminal neuralgia, the recurrence rates in patients with arterial and venous compressions were high in our series. Considering that our patients with simple venous compression had good surgical outcomes, the importance of the complete 
decompression should be emphasized, as discussed in the section on surgery for trigeminal neuralgia.

\section{Conclusions}

The dissection of venous systems is important in microvascular decompression surgery for trigeminal neuralgia and hemifacial spasms. Preservation of the superior petrosal vein may be useful for reducing the risk of venous congestion. Cutting the arachnoid sleeve that envelops the superior petrosal vein usually helps to safely open the surgical corridors to the trigeminal nerve. Patients with venous compression of the root of the trigeminal and facial nerves may have differences in their cure and recurrence rates, compared to patients with arterial compression. Further prospective surgical investigations with elaborated MR-based neuroimaging may precisely clarify the clinical significance of venous compression in the future.

\section{References}

1. Burchiel KJ: A new classification for facial pain. Neurosurgery 53:1164-1167, 2003

2. Campos-Benitez M, Kaufmann AM: Neurovascular compression findings in hemifacial spasm. J Neurosurg 109:416-420, 2008

3. Cheng L: Complications after obliteration of the superior petrosal vein: are they rare or just underreported? J Clin Neurosci 31:1-3, 2016

4. Dumot C, Brinzeu A, Berthiller J, Sindou M: Trigeminal neuralgia due to venous neurovascular conflicts: outcome after microvascular decompression in a series of 55 consecutive patients. Acta Neurochir (Wien) 159:237-249, 2017

5. Dumot C, Sindou M: Trigeminal neuralgia due to neurovascular conflicts from venous origin: an anatomical-surgical study (consecutive series of 124 operated cases). Acta Neurochir (Wien) 157:455-466, 2015

6. Feng BH, Zheng XS, Wang XH, Ying TT, Yang M, Tang YD, et al: Management of vessels passing through the facial nerve in the treatment of hemifacial spasm. Acta Neurochir (Wien) 157:1935-1940, 2015

7. Han IB, Chang JH, Chang JW, Huh R, Chung SS: Unusual causes and presentations of hemifacial spasm. Neurosurgery 65:130-137, 2009

8. Hong W, Zheng X, Wu Z, Li X, Wang X, Li Y, et al: Clinical features and surgical treatment of trigeminal neuralgia caused solely by venous compression. Acta Neurochir (Wien) 153:1037-1042, 2011

9. Ikeda N, Toda H, Yamamoto M, Kanemaru S, Ishikawa M, Iwasaki K: A perforating artery compressing the nerve rootlet and causing glossopharyngeal neuralgia. Neurosurgery 11 (Suppl 3):382-386, 2015

10. Inoue T, Hirai H, Shima A, Suzuki F, Fukushima T, Matsuda M: Diagnosis and management for trigeminal neuralgia caused solely by venous compression. Acta Neurochir (Wien) 159:681-688, 2017

11. Lee SH, Levy EI, Scarrow AM, Kassam A, Jannetta PJ: Recurrent trigeminal neuralgia attributable to veins after microvascular decompression. Neurosurgery 46:356-362, 2000

12. Liebelt BD, Barber SM, Desai VR, Harper R, Zhang J, Parrish R, et al: Superior petrosal vein sacrifice during microvascular decompression: perioperative complication rates and comparison with venous preservation. World Neurosurg 104:788-794, 2017

13. Masuoka J, Matsushima T, Hikita T, Inoue E: Cerebellar swelling after sacrifice of the superior petrosal vein during microvascular decompression for trigeminal neuralgia. J Clin Neurosci 16:1342-1344, 2009

14. Matsushima K, Matsushima T, Kuga Y, Kodama Y, Inoue $\mathrm{K}$, Ohnishi $\mathrm{H}$, et al: Classification of the superior petrosal veins and sinus based on drainage pattern. Neurosurgery 10 (Suppl 2):357-367, 2014

15. Matsushima T, Huynh-Le P, Miyazono M: Trigeminal neuralgia caused by venous compression. Neurosurgery 55:334-339, 2004

16. McLaughlin MR, Jannetta PJ, Clyde BL, Subach BR, Comey $\mathrm{CH}$, Resnick DK: Microvascular decompression of cranial nerves: lessons learned after 4400 operations. J Neurosurg 90:1-8, 1999

17. Miller JP, Acar F, Burchiel KJ: Classification of trigeminal neuralgia: clinical, therapeutic, and prognostic implications in a series of 144 patients undergoing microvascular decompression. J Neurosurg 111:1231-1234, 2009

18. Pathmanaban ON, O'Brien F, Al-Tamimi YZ, HammerbeckWard CL, Rutherford SA, King AT: Safety of superior petrosal vein sacrifice during microvascular decompression of the trigeminal nerve. World Neurosurg 103:84-87, 2017

19. Rhoton AL Jr: The cerebellopontine angle and posterior fossa cranial nerves by the retrosigmoid approach. Neurosurgery 47 (3 Suppl):S93-S129, 2000

20. Rhoton AL Jr: The posterior fossa veins. Neurosurgery 47 (3 Suppl):S69-S92, 2000

21. Toda H, Goto M, Iwasaki K: Patterns and variations in microvascular decompression for trigeminal neuralgia. Neurol Med Chir (Tokyo) 55:432-441, 2015

22. Xia L, Liu MX, Zhong J, Dou NN, Li B, Sun H, et al: Fatal complications following microvascular decompression: could it be avoided and salvaged? Neurosurg Rev 40:389-396, 2017

23. Zacest AC, Magill ST, Miller J, Burchiel KJ: Preoperative magnetic resonance imaging in Type 2 trigeminal neuralgia. J Neurosurg 113:511-515, 2010

\section{Disclosures}

The authors report no conflict of interest concerning the materials or methods used in this study or the findings specified in this paper.

\section{Author Contributions}

Conception and design: Toda. Acquisition of data: Toda, Yoshimoto, Miki, Hashikata, Goto, Nishida. Analysis and interpretation of data: Toda. Drafting the article: Toda. Critically revising the article: Toda, Iwasaki, Goto. Reviewed submitted version of manuscript: Toda, Iwasaki, Yoshimoto, Miki, Hashikata, Nishida. Approved the final version of the manuscript on behalf of all authors: Toda. Statistical analysis: Toda. Study supervision: Toda.

\section{Supplemental Information \\ Videos}

Video 1. https://vimeo.com/268382467.

\section{Correspondence}

Hiroki Toda: Fukui Red Cross Hospital, Fukui, Japan. htoda-nsu@umin.ac.jp. 